\title{
AN OPERATION FOR THE TREATMENT OF SEVERE CONTRACTION OF THE SOCKET*
}

\author{
BY \\ B. W. RYCROFT \\ From the Corneo-Plastic Unit and Regional Eye Bank, Queen Victoria Hospital, \\ East Grinstead, Sussex \\ "The restoration of an orbital socket is one of the most difficult tasks an ophthalmic surgeon has to perform"
Spaeth
}

TOWARDS the end of the 19th century, excision of the eye was practised far more frequently than it is to-day: London streets had their full quota of old soldiers or beggars who had lost one or both eyes. A sense of aesthetic outrage caused Streatfeild (1872) to write:

"Some patients again are met with, whether in private or hospital practice, who either before or since the excision operation, are quite incapable, and cannot by any surgical method be made capable of wearing an artificial eye even if they desire it; such are the cases of irregular cicatrization within the lids caused by wounds or burns or ill surgery and producing obstinate entropion, strong cicatricial bands in any direction, or a narrowing of the whole conjunctival sac or of the palpebral aperture by any of the above means."

Streatfeild's method of treating this contraction was to excise as much of the conjunctiva as he could reach and to cauterize the remainder with zinc chloride. He also advised that the interpalpebral space should be obliterated at the time of enucleation.

Green (1884) excised the lid margins and lashes together with the conjunctiva and then sutured the lid margins together to get a continuous cutaneous surface. These operations were done at the same time as the enucleation of the eyeball since in this period the manufacture of adequate prostheses was still in its infancy.

Yet to-day there is still a place for this type of operation in modern ophthalmic surgery, and at East Grinstead it has had to be revived for the final treatment of severe contraction of the socket. Such a melancholy condition results from the irritation caused by an unsuitable artificial eye which has been worn over the years and followed by infection and contraction, or from repeated surgical attempts at the reconstruction of the socket by free grafts after severe burns, wounds, or civil injuries.

For the patient severe contraction of the socket is a social embarrassment because it prevents the use of a satisfactory artificial eye as a cosmetic improvement, and it is also a source of constant annoyance because of the unpleasant discharge and irritation. For the surgeon contraction of the socket

* Received for publication December 1, 1960. 
is often a technical temptation which all too frequently ends in disappointment to the patient. The true assessment of any cosmetic operation is the final opinion of the patient and not that of the surgeon.

For the moderate and localized degrees of fornix deficiencies much can be repaired by the use of free mucous membrane grafts from the mouth or conjunctiva, and if this is combined with a delayed implant in Tenon's capsule, a tolerable cosmetic improvement for an artificial eye may be obtained. A total lining to the socket is a very different problem, requiring not only prolonged hospitalization but also constant aftercare to keep the socket clean and wholesome. Mucous membrane linings constantly ooze and give rise to a discharge with varying degrees of chronic infection; on the other hand the skin linings may remain dry but in the course of time an unpleasant odour nearly always develops. A combination of the two tissues is sometimes used, but this is an unhappy marriage because the secretion from the mucous membrane component of the combined graft generally causes excoriation of the skin portion with resultant discomfort and an unpleasant smell. In any case the end-result of total socket reconstruction is never really entirely satisfactory from the cosmetic point of view, since the artificial eye maintains a fixed stare and is generally covered by inspissated discharge.

The limit of economic tolerance by the patient and of the enthusiasm of the surgeon is reached when various types of reconstructive surgery by means of grafts have persistently failed and when there is nothing further to offer except a repetition of the whole procedure. It is then that total excision of the lining of the socket resulting in permanent closure offers complete relief, and when this is combined with the modern application prosthesis the cosmetic result is satisfactory.

Total excision of the socket offers a far better cosmetic and surgical result than can ever be obtained by total reconstruction, graft by graft and week by week of hospitalization, when severe contraction of the socket is present. By removing all the conjunctiva as well as the lacrimal gland the sources of moisture and discharge are eliminated. A smooth skin surface remains which requires no after care, and this is a boon which is much appreciated by patients who have spent years washing out their sockets, cleaning their eyes, day in and day out. The prosthesis can now be made as a perfect match for the other eye and is quite frequently undetectable, which is more than can be said for the artificial eye in a contracted socket which has suffered inadequate reconstruction. In these severe cases movement of the artificial eye can never be obtained to any satisfactory degree, but when the patient wears a matched ophthalmic prosthesis he quickly learns to hide the fact of immobility by turning his head rather than his eye.

\section{Technique of the Operation}

For this type of surgery the absence of bleeding is a great advantage and at East Grinstead hypotensive anaesthesia has been a routine procedure for many years, 
subject to the guidance of the anaesthetist. The surgical field of operation is rendered practically bloodless and rapid healing is promoted by the absence of haematomata. Generally the systolic blood pressure is maintained at a level of between $50-60 \mathrm{~mm}$. $\mathrm{Hg}$ during the operation and is frequently combined with a postural tilt of the patient of to $30^{\circ}$.

The line of incision which is first marked out along the lid margins runs $2 \mathrm{~mm}$. above or below the lash-bearing area (Fig. 1). It extends along the outer canthus which is split outwards for $4 \mathrm{~mm}$. and at the inner canthus the incisions close to include the lacrimal canaliculi. The lid margins and lashes are then completely excised in two strips and the conjunctiva is freed from the underlying lids and dissected well back; an attempt is always made to excise the entire conjunctival cul-desac in one piece, but this is not always possible if there have been old grafts or if deep cicatricial bands run back to the apex of the orbit. Care should be taken to excise all conjunctiva completely without disturbing the deeper structures of the orbit. Since the socket is generally already markedly contracted, the area to be excised is not large. Next, the lateral edges of the wound are widely retracted and the lacrimal gland is exposed, mobilized, and completely excised. If this is not done and a piece of lacrimal gland is allowed to remain a sinus will result, and in one case secondary excision of gland tissue had to be performed. It is the author's opinion that the lacrimal gland should always be excised as a routine procedure in all cases of formal enucleation, because in the absence of an eyeball the protective function of the lacrimal secretion is no longer required, and the drainage of a socket is in any case completely altered and made inadequate by removal of the globe There remains sufficient moisture in the normal conjunctiva to lubricate an artificial eye and any excess moisture only collects and encourages stagnation in the socket with risk of subsequent infection.

FIG. 1.-The line of incision includes the inner and outer canthus as well as the lashbearing areas of both lids in all thicknesses.

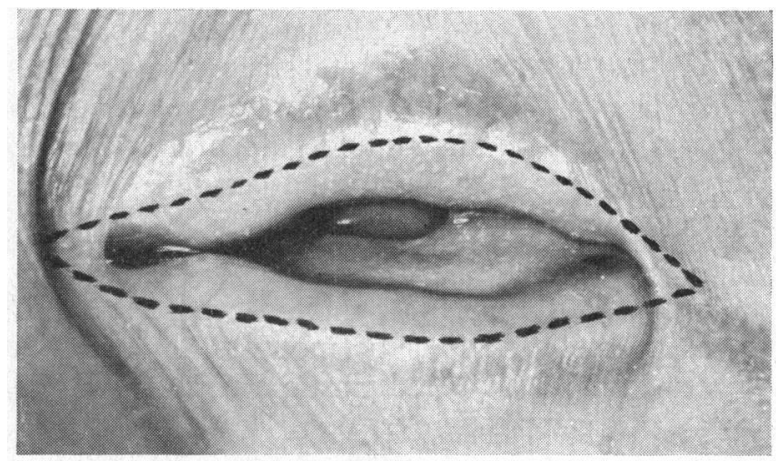

The skin edges of the upper and lower lids are now brought together and carefully stitched by interrupted silk stitches; at intervals pressure is put on the socket to expel any accumulation of blood. A paraffin gauze dressing is applied together with a firm built-up pressure bandage, which is not disturbed for 4 days. Pressure is maintained for 7 days and the stitches are removed on the 10th day. Usually a smooth lax skin surface is now seen with a hair line suture junction, and at the end of a fortnight the patient is ready for the technician to fit a prosthesis. 
Such a prosthesis may be mounted on a spectacle frame which holds it closely against the skin, or it may be stuck to the skin by Mastisol or some other suitable adhesive without the use of spectacle frames.

\section{The Prosthesis}

Mr. H. S. Warren, of Messrs. Clement Clarke Ltd., is responsible for the orbital prostheses which are used at East Grinstead and he has kindly supplied the following details of his technique: "Given an orbital depression sufficiently deep for the plastic restoration to be orientated correctly, the prosthetist with experience of moulding, painting, carving, and the making of imitation eyes, can construct a prosthetic eye and eyelids in pigmented methylmethacrylate plastic material that will be durable and almost undetectable. The majority of my orbital prostheses are stuck on with a film of gum mastic solution, thinly applied to the fitting area of the prosthesis. After removal at night, the old gum is "cleaned off" with ether solvents and the anterior surface may be cleaned with a small brush, detergent, and water. Gum attachment is contraindicated for elderly, partially sighted, and blind patients, and also in certain skin conditions. For these cases spectacles can be used for retention: the prosthesis may be permanently attached to the frames or, when the nasal bridge is well defined and the orbital depression provides a shelf, one small flat wire spring may extend from the spectacle side and another from the bridge to marry with grooves formed in the prosthesis. The patient should be measured for the spectacles when the prosthesis is at the trial stage in wax, so that a compromise can be made in the respective designs in order to achieve the best support and cosmetic effect.

To construct the prosthesis, a stone model is made from an alginate impression of the upper half of the face. The pattern eye is prepared in white wax and the iris and cornea are inserted. The position is ascertained by measurement and by trial and error. The lids and orbital surround are shaped and sculptured in pink dental wax. The combination of eye and lids is tried on the patient and modified until the eyes, catoptric images and palpebral fissures are correctly balanced, with the patient in a relaxed position and gazing straight ahead.

The eye and surround are separately invested in a double or multi-part mould, which is then packed with monomer-polymer dough, opacified, and pigmented for the basic shade. Separate mixes of more concentrated colours are worked in to represent the variations of mottle. Pigments in acrylic solution can also be applied to the basic cured plastic which is then dried in an oven and finished with a layer of clear plastic over it."

\section{Case Reports}

Case 1, a male patient, had been unable to wear an artificial eye for several years owing to severe contraction of the socket, and although many special-shaped eyes had been tried the eye always fell out. The fornices were irregular and shallow and there was a persistent discharge which was a considerable annoyance at work. Fig. 2(a) shows the pre-operative condition, Fig. 2(b) the condition on discharge from hospital, Fig. 2(c) the spectacle prosthesis, and Fig. $2(d)$ the prosthesis in situ. 


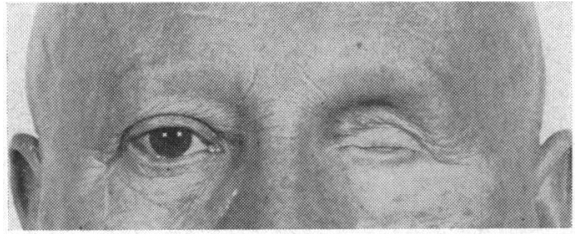

FIG. 2a.-Severe contraction of socket before surgery.

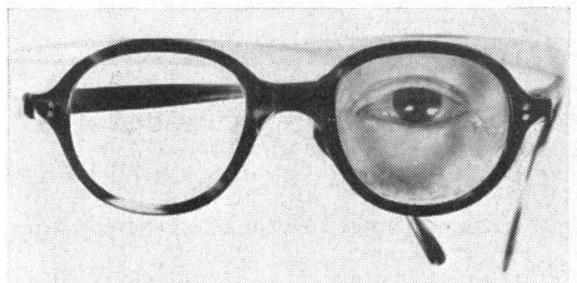

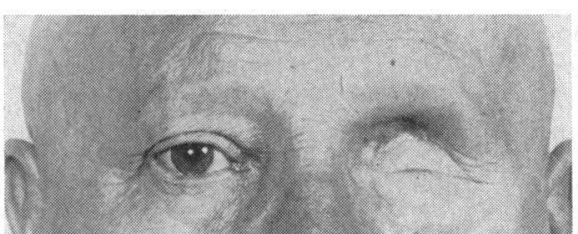

FIG. 2b.-Appearance after complete excision of socket and lacrimal gland.

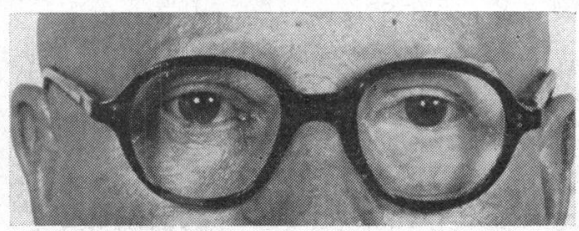

FIG. 2d.-Spectacle prosthesis in situ.

FIG. $2 c$.- Spectacle prosthesis detached.

Case 2, a male patient, had had two implants in the left eye after enucleation (one a buried implant with muscle attachments and the other a free globe in Tenon's space), and both had been extruded. The excessive contraction of the socket in this case was due to unsuspected chronic pemphigus. A small symblepharon was noted in the inferior conjunctival sulcus of the other eye and on inquiry it was stated that there had been a vesicular skin eruption some years previously. Further attempts to reconstruct this socket would have inevitably been followed by the severe contraction which usually follows conjunctival surgery in most forms of ocular pemphigus.

Case 4, a boy aged 8 yrs, shows the late results of socket contracture after radiation for bilateral gliomata. The left eye is artificial (Fig. 3a). The right socket was excised and a spectacle prosthesis fitted (Fig. $3 b$ ).

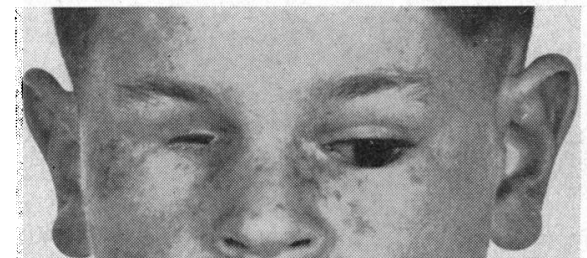

Fig. 3a.-Enucleation of both eyes for bilateral gliomata with radiation. Marked contraction of right socket.

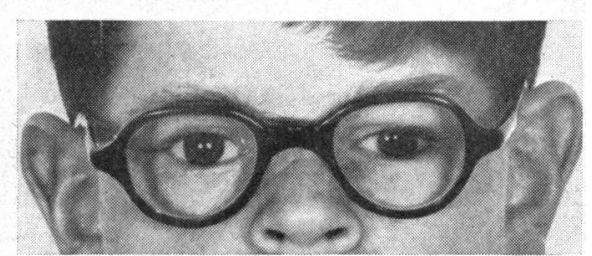

FIG. 3b.-The right socket has been excised and a spectacle prosthesis is in place.

Case 5, a man aged 58 yrs, had suffered severe chemical burns. On the left side was a shrunken globe with obliteration of the fornices and severe irritation with discharge. On the right side, since there was perception of light in the eye, reconstruction of the fornices had been undertaken to free the lids from the globe. Fig. 4(a) shows the left socket before excision, Fig. 4(b) the excision, and Fig. 4(c) the prosthesis in situ (Fig. 4a-c, overleaf).

A better cosmetic result could be obtained in this case by excising the right socket and globe also as there is no possibility of any restoration of sight. However, perception of light alone means so much to a blind person that no further surgery is contemplated, at least for the time being. 
(a)

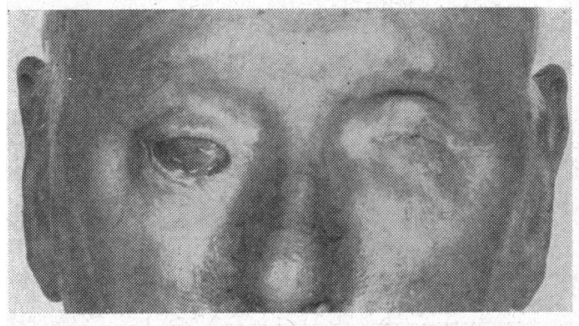

(c)

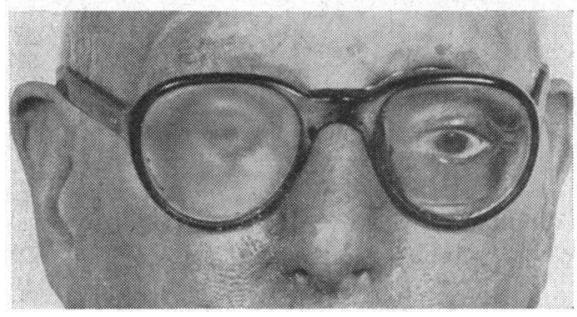

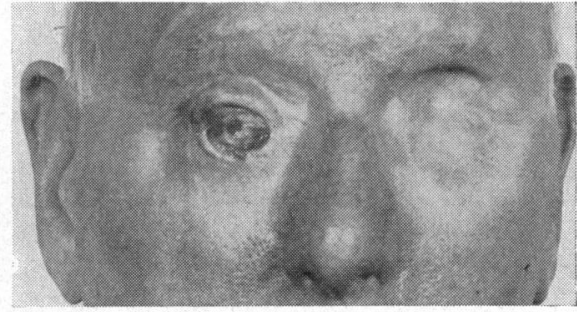

FIG. 4a,-Severe contraction of left socket after chemical burns.

FIG. 4b.-Appearance after excision of left socket and lacrimal gland.

FIG. 4c.- Spectacle prosthesis in situ. The right socket will not be excised as there is bare perception of light.

Case 6, a female aged 66, had worn an artificial eye for 10 years after enucleation in adolescence, but had since been unable to obtain an eye which could be retained. The upper fornix was of irregular depth, being deep in the outer half but almost absent in the inner half, and the lower fornix was completely obliterated. At her advanced age it was considered that excision of the socket would give speedy relief and that extensive reconstruction of the fornices was not desirable. The patient was in hospital for only 8 days and is entirely satisfied with the result.

\section{Conclusions}

The advantages of this method of treatment are as follows:

(1) The stay in hospital is short and rarely exceeds 10 days.

(2) No after-care is needed as the smooth skin surface gives no trouble.

(3) There is no discharge, discomfort, or irritation.

(4) The surgical technique is very simple.

(5) Every patient has expressed satisfaction with the operation, not only because of the cosmetic result but also because of the relief after months of irritation and tedious palliative measures.

As Streatfeild continued .... "It is a disgrace to surgery not to close up the ugly chasm and it is easily done".

Mr. H. S. Warren of Messrs. Clement Clarke Ltd., London is responsible for the prosthetic appliances used at the Corneo-Plastic Unit and Regional Eye Bank, East Grinstead; it is a pleasure to acknowledge his enthusiastic co-operation and skill in the treatment of these cases.

Mr. Gordon Clemetson, Director of Photography, has kindly provided the photographs, and I am also indebted to Dr. Anthony Edridge and Messrs. Werb, Griffiths, and Cowan for their valuable assistance.

\section{REFERENCES}

GreEN J. (1884). Amer. J. Ophthal., 1, 65.

STREATFEILD, J. F. (1872). Lancet., 2, 849. 